\title{
Análise Histológica e Histoquímica de Fatores Prognósticos em Pacientes com Retocolite Ulcerativa
}

\author{
Histological and Histochemical Analysis of Prognostic Factors \\ in Patients with Ulcerative Colitis
}

\author{
GEORGE LUIZ DE SOUZA ARAÚJO ${ }^{1}$; ADRIANA MARIA DA SILVA TELLES ${ }^{2}$; \\ FRANCISCO EDUARDO DE ALBUQUERQUE LIMA ${ }^{3}$; NICODEMOS TELES DE PONTES FILHO ${ }^{4}$; \\ MARCOS CÉSAR FEITOSA DE PAULA MACHADO ${ }^{5}$ \\ ${ }^{1}$ Mestre em Patologia; ${ }^{2}$ Doutora em Nutrição; ${ }^{3}$ Doutor em Cirurgia $;{ }^{4}$ Doutor em Nutrição; ${ }^{5}$ Mestre em Patologia. \\ Todos da Universidade Federal de Pernambuco.
}

\begin{abstract}
ARAÚJO GLS; TELLES AMS; LIMA FEA; PONTES FILHO NT; MACHADO MCFP. Análise Histológica e Histoquímica de Fatores Prognósticos em Pacientes com Retocolite Ulcerativa. Rev bras Coloproct, 2009;29(1): 007-014.

RESUMO: Objetivo: Visando oferecer um método que auxilie o diagnóstico diferencial ou mesmo que seja indicador da transformação neoplásica, a galectina-3 surge como um potencial marcador da fisiopatologia de diversos tipos de câncer, entre eles o câncer colorretal. Métodos: o perfil imuno-histoquímico foi obtido do tecido intestinal de pacientes com retocolite ulcerativa $(\mathbf{n}=20)$ e sua respectiva contraparte normal, de ambos os sexos e idade média de 55 anos. Os tecidos foram submetidos à histoquímica da reação de Schiff (PAS) para visualizações das inclusões de glicosaminoglicanos e tricrômico de Masson para visualização das fibras colágenas. Fragmentos de tecido $(4 \mu \mathrm{m})$ foram submetidos à técnica de imuno-histoquímica para a proteína galectina-3. A marcação tecidual foi analisada através de uma estação de trabalho, contendo um microscópio óptico equipado com uma câmera digital, ambos acoplados a um computador contendo o software OPTIMAS ${ }^{\circledast}$. Resultados: Os resultados demonstram uma diminuição da expressão da galectina-3 no sitio inflamatório da retocolite ulcerativa. Houve um aumento significativo $(\mathbf{p}<\mathbf{0 . 0 2 2})$ no número de células caliciformes dos pacientes tratados clinicamente, porém com redução do conteúdo glandular. Conclusões: Os dados sugerem que a atividade glandular juntamente com a redução da expressão da galectina-3 possam auxiliar o diagnóstico e o monitoramento desses pacientes.
\end{abstract}

Descritores: Retocolite ulcerativa, galectina-3, análise digital de imagens.

\section{INTRODUÇÃO}

A retocolite ulcerativa idiopática (RCUII) e a doença do Crohn (DC) são as formas mais comuns das doenças inflamatórias intestinais (DII), que se caracterizam por inflamação crônica do intestino, de etiologia ainda não definitivamente esclarecida e em cuja patologia estão envolvidos fatores genéticos,

Trabalho realizado no Laboratório de Imunopatologia Keizo Asami (LIKA)/UFPE.

$\overline{\text { Recebido em 15/10/2008 }}$

Aceito para publicação em 27/11/2008 
Análise Histológica e Histoquímica de Fatores Prognósticos

em Pacientes com Retocolite Ulcerativa

George Luiz de Souza Araújo e Cols. ambientais e imunológicos (1). As DII ocorrem em todo o mundo e representam sério problema de saúde, pois atingem preferencialmente pessoas jovens, cursam com recidivas frequentes e admitem formas clínicas de alta gravidade (2). A DII é resultante de ativação persistente e inadequada do sistema imune mucoso (3), admitindo-se que possa estar relacionada com um distúrbio autoimune (4).

Levando-se em consideração os fatores genéticos, é sabido que a retocolite é um fator predisponente ao surgimento de câncer de cólon (5). Na RCUI, são reconhecidos três tipos de lesões pré-malignas: a displasia plana, as lesões/tumores associados à displasia e os pólipos adenomatosos esporádicos (6).

Neste sentido Rutter e colaboradores (7) dão ênfase ao processo inflamatório estabelecido em longo prazo, como sendo fator determinante da carcinogênese colorretal envolvida na DII. Contudo, existem indícios de que a carcinogênese oriunda da inflamação crônica poderia estar relacionada com um dano causado pela ativação prolongada das vias de sinalização responsáveis pela renovação celular continuada (8). Apesar destes achados, um estudo retrospectivo (9) demonstrou que a severidade da inflamação é o fator que ofereceria maior risco para o surgimento de uma neoplasia, com destaque, para o tempo da doença e para a extensão da mesma (pancolite).

As diferenças regionais com relação à frequência, à apresentação clínica e à gravidade tanto da DC quanto da RCUI, podem provavelmente ser explicadas por fatores de natureza genética e ambiental (10). Outro aspecto que deve ser levado em consideração no estudo das DII é a grande escassez de estudos sobre a ocorrência da DC e da RCUI nos países em desenvolvimento (11) o que inclui, sobretudo, o Brasil. Dessa maneira tornando mais difícil a realização de inquéritos epidemiológicos sobre estas doenças.

\section{Imunobiologia da RCUI}

O epitélio intestinal é composto por uma camada simples polarizada recoberto por muco, no qual as bactérias comensais estão mergulhadas. Existem relatos na literatura de que defeitos na produção de muco são descritos em pacientes com RCUI (12).

Por sua vez, uma população de enterócitos altamente especializada serve como interface entre a camada epitelial e o tecido linfóide subjacente - as células dentríticas. Estas células são a chave no controle da imunidade contra patógenos bem como a tole- rância com os microorganismos comensais, além de expressarem uma série de moléculas receptoras capazes de fazer a distinção entre comensais e patógenos, com isso, ativando ou silenciando respostas mediadas por células $\mathrm{T}$ (13).

Atualmente já está bem estabelecido que a doença inflamatória intestinal resulte de uma resposta inapropriada de um sistema imune de mucosa defeituoso incapaz de distinguir a microbiota de outros antígenos luminais (14) Mas, o modo e o porquê de como esta indução inapropriada acontece ainda esta sendo alvo de pesquisas.

\section{Patologia geral da RCUI}

Na prática clínica o termo doença inflamatória intestinal é usualmente reservado para a RCUI, para DC e para a DII de tipo indeterminado. A RCUI é uma doença inflamatória crônica que envolve primariamente o reto e intestino grosso. Ela acomete o reto e se estende proximalmente de modo contíguo, podendo variar desde uma proctite a uma pancolite dependendo do grau de atividade da doença. (15)

Em relação às características histológicas da RCUI, a avaliação da arquitetura tecidual, bem como da natureza do infiltrado inflamatório indicam se o processo visualizado é crônico ou idiopático, que na RCUI a característica principal é a distorção da arquitetura tecidual do revestimento mucoso do cólon. A literatura aponta que os pacientes com RCUI têm um risco mais alto de desenvolver câncer colorretal do que a população geral (16). A extensão e a duração da doença são dois fatores críticos que corroboram esta associação, apesar de estudos recentes também apontarem que a gravidade da doença é mais um fator predisponente ao surgimento da neoplasia intestinal. Para diminuir este risco, os pacientes são incluídos em programas de vigilância, com realização colonoscópica com biópsias seriadas dos segmentos do cólon e reto anuais naqueles cuja doença é diagnosticada entre 8 e 10 anos.

Mesmo com todas essas informações a respeito do comportamento dessas doenças, existe necessidade de novos métodos para aperfeiçoar o diagnóstico de fatores de risco adicionais para a progressão neoplásica, permitindo o acompanhamento de pacientes com alto risco. Atualmente, aconselha-se que o estudo histopatológico da mucosa retal deve sempre ser realizado, e se constitui no método diagnóstico mais importante, embora não-patognomônico para o esclarecimento da etiologia da colite (17). O diagnóstico 
Análise Histológica e Histoquímica de Fatores Prognósticos

em Pacientes com Retocolite Ulcerativa

George Luiz de Souza Araújo e Cols. histopatológico de amostras teciduais obtidos por endoscopia é essencial e crucial para determinação do tratamento (18).

\section{Galectina-3}

As galectinas pertencem a uma família de proteínas que se ligam a resíduos de galactose em glicoconjugados (glicoproteínas e/ou glicolipídios) e representam um grupo especial das chamadas proteínas ligantes de carboidrato, as lectinas (19). A estrutura molecular das galectinas geralmente consiste em cerca de 130 aminoácidos, altamente conservados, bem como uma região de reconhecimento de carboidratos (CRD - carbohydrate recognition domain) e estruturas de â-galactosídeos (20).

Até o presente, 14 membros da família das galectinas foram identificados, entre eles está a Galectina-3. Esta se encontra distribuída em uma ampla variedade de células e tecidos, podendo ser encontrada no interior da célula ou mesmo secretada no espaço extracelular (20). E um grande número de processos biológicos tanto extracelulares quanto intracelulares envolve galectina-3, refletindo a participação desta proteína em condições fisiológicas e patológicas (20).

Em muitos casos, a expressão das galectinas encontra-se favorecida por fatores de crescimento ou mesmo oncogenes, e atenuada em razão de genes supressores tumorais. Com respeito às propriedades funcionais da galectina-3, esta lectina assume múltiplos papéis na patogênese, proliferação e disseminação de metástases (20), por meio da modulação da adesão das células malignas em sítios distantes através do endotélio vascular (19). Assim, a galectina-3 pode ser encontrada em uma ampla variedade de malignidades $(19 ; 21)$.

\section{Análise digital de imagens em Patologia}

Diferentes métodos de análise têm sido aplicados com sucesso para traduzir de forma mais objetiva, e numericamente representativa as transformações que ocorrem nas células tumorais e teciduais (22), seja através da análise colorimétrica de células neoplásicas em cultura, da citometria automática ou das variações morfométricas nucleares (23).

Os resultados obtidos pela histoquímica de células tumorais contam com o apoio dos sistemas computadorizados de análise de imagens, os quais fornecem dados qualitativos e quantitativos mais refina- dos esclarecendo assim, vários aspectos histomorfológicos das transformações neoplásicas (24). Nesse sentido a análise digital de imagens tem sido de grande importância para o diagnóstico, especialmente nos casos onde a suspeita clínica de câncer prevalece mesmo após uma biópsia negativa (25).

Assim, nos últimos anos tem-se acentuado o interesse no diagnóstico diferencial da DC e da RCUI, DIIs que apresentam aspectos epidemiológicos e clínicos comuns. Entretanto, diferem de modo significativo quanto à evolução, à forma de exteriorização clínica e à resposta ao tratamento, permitindo caracteriza-las como entidades distintas. Por este motivo a utilização de ferramentas moleculares como apoio ao diagnóstico vem ganhando espaço no sentido de identificar marcadores celulares específicos.

A partir daí, a expressão de galectina-3 tem emergido como um potencial marcador diagnóstico/ prognóstico de algumas neoplasias e condições inflamatórias diversas. Nesse contexto, o presente trabalho propôs-se a buscar uma correlação entre a expressão tecidual da galectina- 3 de pacientes com retocolite ulcerativa, juntamente com marcadores histoquímicos para obter dados quantitativos e qualitativos que possam auxiliar no prognóstico das doenças inflamatórias intestinais.

\section{METODOLOGIA}

\section{Seleção de Casos}

Os critérios de inclusão dos pacientes foram: diagnóstico clínico acima de 7 anos baseado em sintomas, radiologia ou colonoscopia, apresentação de displasia no momento do exame. Já aqueles pacientes que foram submetidos a algum processo cirúrgico do cólon (plástica ou remoção de tumores), portadores de doença de Crohn ou colite indeterminada foram excluídos do estudo.

Foram escolhidos blocos de parafina, contendo fragmentos de tecido de intestino anteriormente diagnosticados com retocolite ulcerativa $(n=20)$ dos arquivos do setor de patologia do LIKA, órgão suplementar da Universidade Federal de Pernambuco. Para controle, foi utilizada a contraparte normal das bordas das amostras sem alterações de natureza anatômica ou funcional.

A partir daí os blocos foram divididos em dois grupos: blocos de pacientes tratados clinicamente $(n=12)$ e blocos de pacientes tratados cirurgicamente 
Análise Histológica e Histoquímica de Fatores Prognósticos

em Pacientes com Retocolite Ulcerativa

George Luiz de Souza Araújo e Cols. $(\mathrm{n}=08)$.

O protocolo experimental desenvolvido no presente trabalho foi aprovado pelo comitê de ética de pesquisas envolvendo seres humanos da Universidade Federal de Pernambuco (CCS/UFPE - protocolo ${ }^{\circ}{ }^{198 /}$ 08).

\section{Imuno-histoquímica para galectina-3}

Foi testada a imunoreatividade da anti-galectina3 conjugada a peroxidase (Sigma, USA) utilizando o protocolo desenvolvido por Hsu e colaboradores (26). Os tecidos foram desparafinizados em xilol e hidratados em álcool (70-100\%). As lâminas com tecidos $(4 \mu \mathrm{m})$ foram incubadas com tampão citrato à $100^{\circ} \mathrm{C}$ por 30 min sob vapor úmido, e em seguida lavadas (por $5 \mathrm{~min}$ ) com PBS e incubadas em leite Molico ${ }^{\circledR}$ (a fim de evitar reações cruzadas), em seguida incubadas com peróxido de hidrogênio por 5 min e lavadas normalmente com PBS, sendo incubadas com uma solução contendo anticorpo monoclonal anti-galectina-3 (na diluição $1: 400)$ por $30 \mathrm{~min}$, em temperatura ambiente $\left(25^{\circ}\right.$ C), em seguida os tecidos foram incubados com anticorpo secundário biotinilado (Kit LSAB DAKO/ USA). A revelação do anticorpo foi obtida através da reação da enzima peroxidase visualizada pela incubação dos tecidos em uma solução contendo diaminobenzidina $\left(\mathrm{DAB}+\mathrm{H}_{2} \mathrm{O}_{2}\right)$. Os cortes foram contracorados com hematoxilina e analisados em microscópio óptico (Olympus BH-2, Japão).

\section{Estudo histoquímico}

Os cortes obtidos foram previamente expostos à rotina histológica e submetidos a diferentes técnicas de coloração: Hematoxilina-Eosina (HE), para observação das características histológicas gerais; e Tricrômico de Masson (TM) e Ácido Periódico de Schiff (PAS) para evidenciar, respectivamente, a deposição de colágeno e a expressão de glicosaminoglicanos. Todos os protocolos experimentais foram desenvolvidos segundo Spicer (27).

\section{Analise Computadorizada de Imagens}

Imagens digitais foram capturadas das lâminas histológicas utilizando um sistema de vídeo-câmera acoplado a um microscópio óptico (Olympus BH-2), já utilizado com sucesso em análises diagnósticas. $\mathrm{O}$ sistema interativo de análise de imagens utiliza o Software OPTIMAS ${ }^{\circledR}$ e Câmera digital CCBBW 410 (Sansung), disponíveis no Departamento de Patologia da UFPE.
Os perfis de marcação dos depósitos PAS+ (Glicosaminoglicanos) numa magnificação de 200x, avaliando-se cinco unidades glandulares por lâmina, perfazendo um total de 60 campos analisados.

Os dados obtidos foram analisados estatisticamente utilizando os testes $t$ de Student, para um nível de significância $(\mathrm{p}<0,05)$.

\section{RESULTADOS E DISCUSSÃO}

As galectinas pertencem a uma família de ligantes de carboidratos extensamente estudadas que estão implicadas em uma ampla variedade de processos biológicos. A característica dessa família de proteínas é possuir um domínio de reconhecimento de carboidratos responsável pela ligação de $\beta$ galactosídeos (21).

No presente estudo, a reação de imunohistoquímica para a galectina- 3 revelou que a proteína foi expressa principalmente nas células epiteliais do cólon, com diferença marcante entre a região normal e a região inflamada; com fraca marcação tanto no grupo tratado cirurgicamente quanto no tratado clinicamente. (Figura 1).

A literatura aponta diversas localizações tanto intracelulares quanto extracelulares para a galectina3. Rubinstein e colaboradores (28) sugerem um papel pró-inflamatório para a galectina-3. Neste sentido, Lippert e colaboradores (29) reforçam esta idéia ao apontarem a galectina-3 como sendo detentora de um potencial papel no comportamento da doença inflamatória intestinal. Isto significa que o processo inflamatório causaria dano às camadas de células epiteliais e estas, por sua vez, poderiam liberar então a galectina3 no espaço intercelular. A galectina-3, dessa forma, estaria conduzindo a uma ativação de outras células, tais como os fibroblastos da lâmina própria colônica, induzindo a um quadro de fibroplasia, como pode ser visualizado na figura 2 .

Por outro lado, pode-se afirmar que já está bem assumido que o risco de desenvolvimento da neoplasia colorretal pode estar relacionado com a inflamação crônica do intestino e que a colite é um importante fator de risco para o câncer colorretal (30).

Isso porque a demasiada exposição aos radicais livres gerados durante o processo de lesão e reparação tecidual poderia causar uma alteração irreversível no ciclo normal de crescimento das células envolvidas 

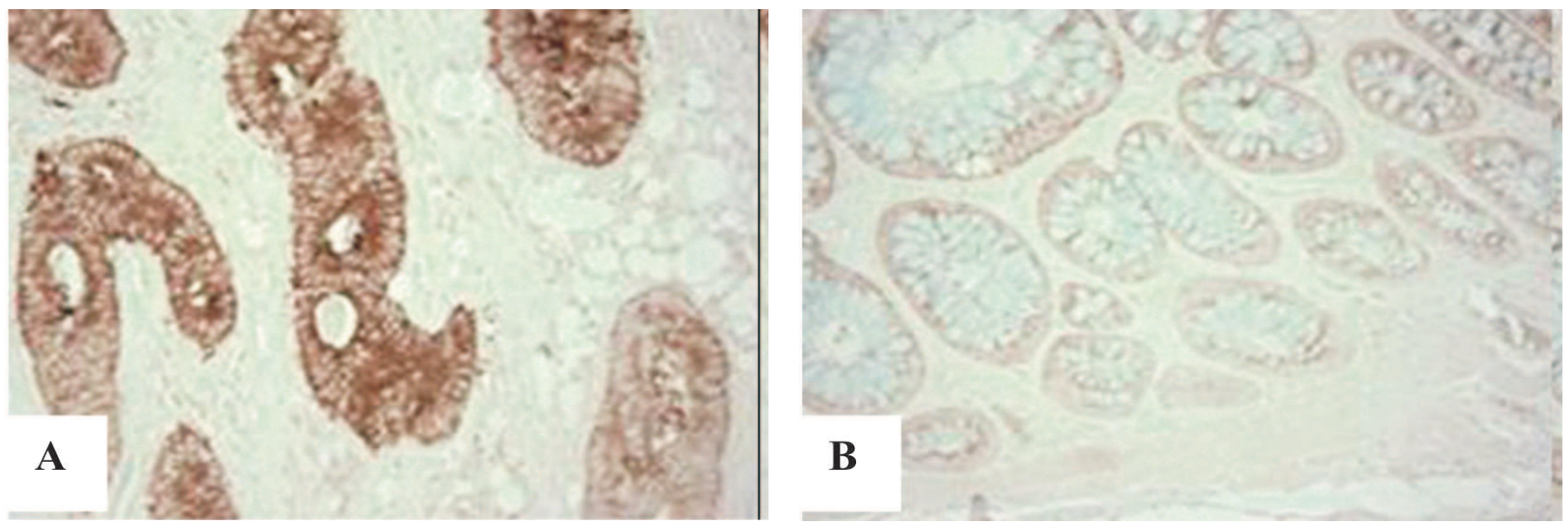

Figura 1 - Perfis de marcação da galectina-3 em tecido colônico em indivíduos sem alterações, mucosa normal com padrão difuso citoplasmático (A). Em B observa-se fraca reatividade em mucosa de paciente com retocolite ulcerativa.(Magnificação, 100x).

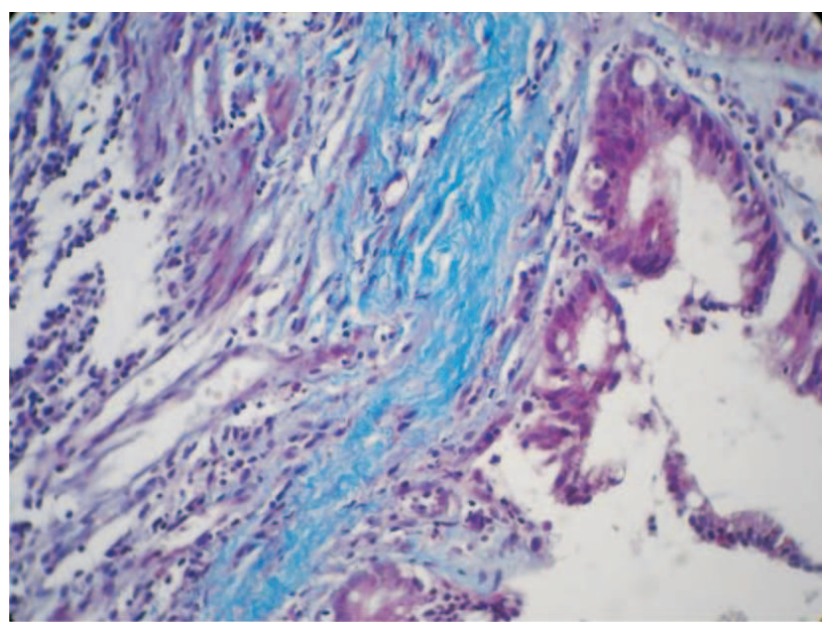

Figura 2 - Fibrose intersticial encontrada na mucosa intestinal de paciente portador de retocolite ulcerativa tratado clinicamente (Tricrômico de Masson, Magnificação 100x).

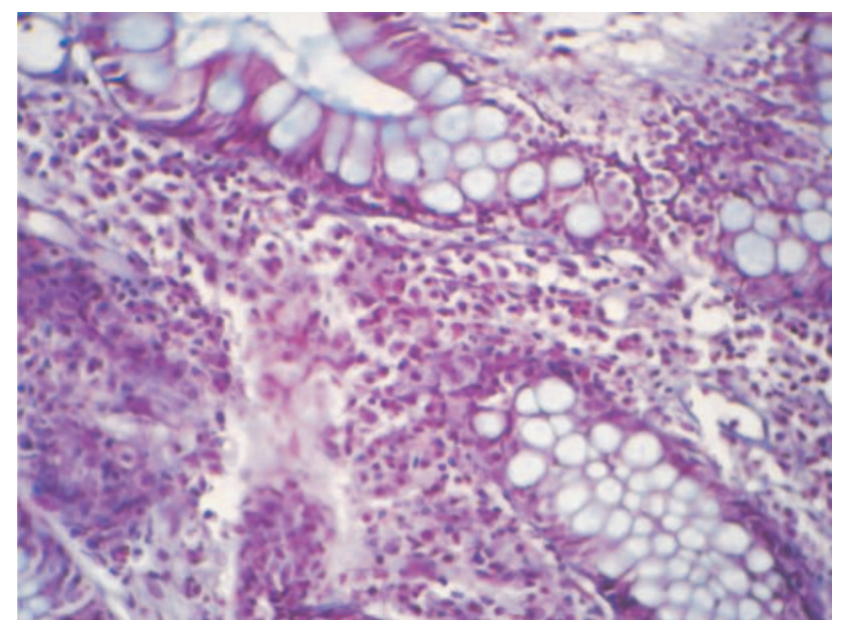

Figura 3 - Área de retocolite ulcerativa evidenciando intenso infiltrado linfoplasmocitário em paciente tratado cirurgicamente (Tricrômico de Masson, Magnificação 100x). no processo. Na figura 3 pode-se observar em pacientes tratados cirurgicamente uma área de tecido apresentando um infiltrado linfoplasmocitário. Além disso, pode-se observar a ausência de fibrose com a presença de raros fibrócitos.

Por outro lado, o padrão de expressão da galectina-3 relacionada à progressão ao câncer colorretal, continua sob debate devido à falta de consenso entre os resultados publicados. Alguns pesquisadores têm encontrado níveis de galectina-3 na progressão ao carcinoma de cólon, enquanto outros têm obtido resultados opostos (31).

Neste presente trabalho, a expressão da galectina-3 foi observada no núcleo e/ou citoplasma nas células de todos os tipos de lesão examinados, bem como na mucosa normal, porém, como dito anteriormente, com diferenças bastante significativas entre os grupos. Vale ressaltar que os nossos resultados indicam que o padrão citoplasmático e nuclear da expressão da galectina-3 não pode ser diretamente relacionado com a progressão ao câncer colorretal, e neste caso poderia assumir diversos significados biológicos, como os descritos acima. Isso porque de todas as amostras analisadas, apenas uma progrediu para a neoplasia colorretal.

Na RCUI ativa, infiltrado neutrofílico, abscessos crípticos e depleção de células caliciformes são achados frequientes (32). Em nosso trabalho, foi observado um infiltrado de plasmócitos basais e linfócitos como indicadores de cronicidade nas lesões estudadas (Figura 3 e Figura 4).

Com o intuito de descrever parâmetros qualitativos e quantitativos, nosso estudo comparou o nú- 


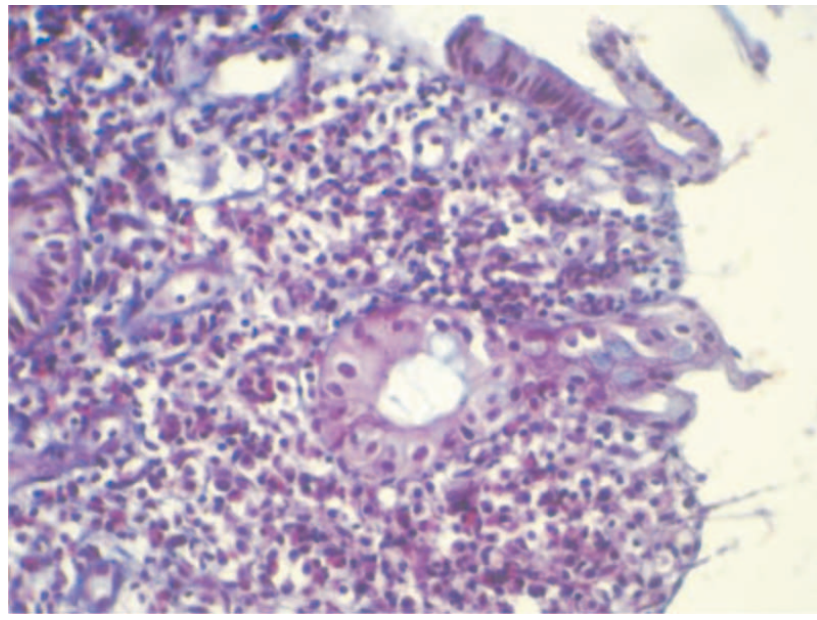

Figura 4 - Área de erosão da mucosa colônica em paciente com retocolite ulcerativa tratado clinicamente (Tricrômico de Masson, Magnificação 100x).

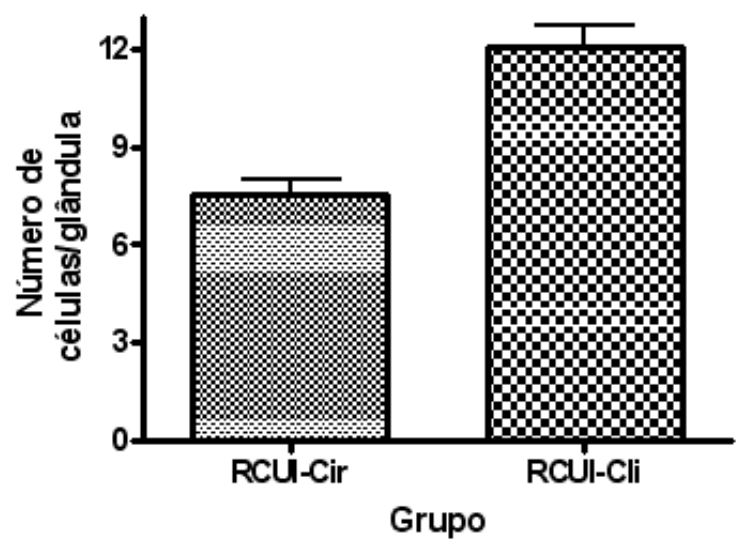

Figura 5 - Comparação entre o número de células caliciformes entre os grupos tratados cirurgicamente (RCUI-Cir) e tratados clinicamente (RCUI-Cli) na retocolite ulcerativa idiopática. ${ }^{*} p<0,022$.

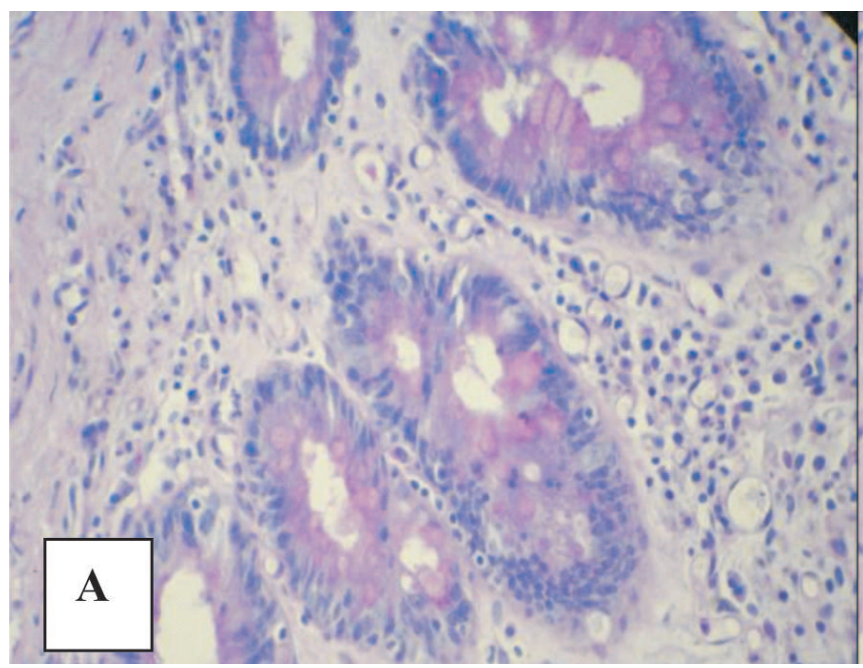

mero de células e a intensidade da marcação histoquímica para glicosaminoglicanos entre dois subgrupos de pacientes da pesquisa. Logo, as amostras dos pacientes tratados cirurgicamente obtiveram uma redução significativa $(\mathrm{p}<0,022)$ do número de células caliciformes com relação ao grupo tratado clinicamente (Figura 5).

Histologicamente observa-se na figura 6 a marcação do PAS mais intensa nos pacientes tratados cirurgicamente, o que reforça a hipótese de que a síntese de muco pelas glândulas intestinais parece estar relacionada à reatividade da doença. Assim, um dado relevante do nosso estudo foi a constatação de que onde há infiltrado inflamatório, existe uma menor produção de muco (Figura 4 e 6A). Logo a submissão desses pacientes a cirurgia para a constituição da bolsa ileal talvez esteja conduzindo à remissão de alguns dos sintomas clínicos observados na retocolite ulcerativa.

A partir dos resultados obtidos, conclui-se que houve redução significativa da expressão tecidual da galectina- 3 em tecido epitelial intestinal de retocolite ulcerativa quando comparado com a contraparte normal de ambos os grupos. Além disso, observou-se um aumento da atividade das células caliciformes em tecido colônico submetido à cirurgia de bolsa ileal, o que poderia indicar o restabelecimento da função normal do tecido. Assim, os dados sugerem que a atividade glandular juntamente com a redução da expressão da galectina-3 possam auxiliar no monitoramento clínico desses pacientes.

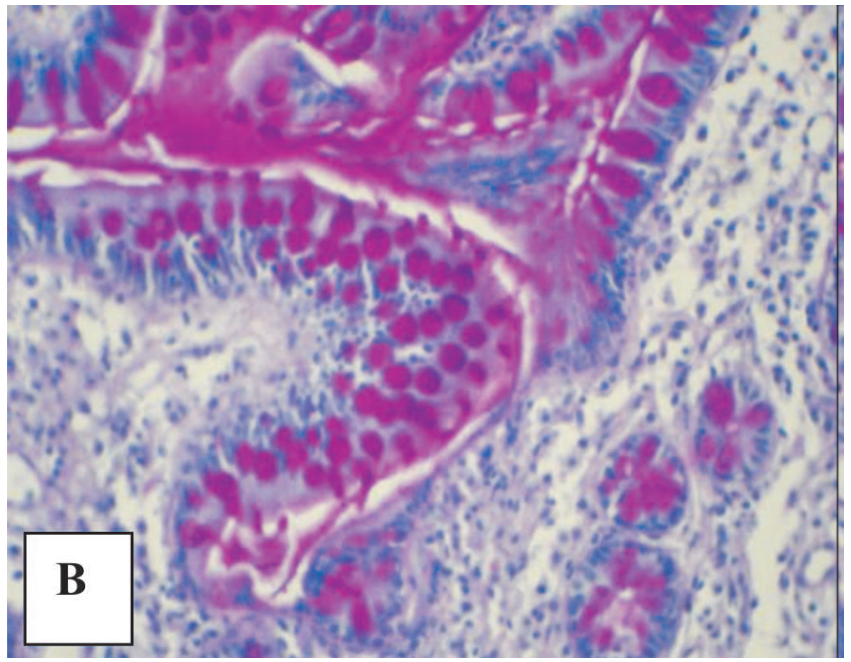

Figura 6 - Marcação PAS de tecido colônico. (A) Os pacientes tratados clinicamente possuem uma menor expressão de glicosaminoglicanos quando comparados com os pacientes tratados cirurgicamente (B). Tricrômico de Masson, Magnificação 100x. 
Rev bras Coloproct Janeiro/Março, 2009
Análise Histológica e Histoquímica de Fatores Prognósticos

em Pacientes com Retocolite Ulcerativa

George Luiz de Souza Araújo e Cols.
Vol. 29

\begin{abstract}
Objectives: Aiming to offer a diagnosis method or a prognostic marker, galectin-3 appears as a potential marker of the physiopathology of diverse diseases and some types of cancer, between them the colorectal cancer. Methods: In our study, the immunohistochemical and histological profiles had been taken from intestinal biopsies of patients with ulcerative colitis ( $\mathrm{n=20}$ ) and its respective normal counterpart, from both males and females and average age of 55 years-old. The biopsies had been submitted to Periodic Acid of Schiff (PAS) for visualization of glycosaminoglycans inclusions, and Masson's tricromic for visualization of collagen fibers. Fragments $(4 \mu \mathrm{m})$ had been submitted to the technique of immunohistochemistry for galectin-3 protein. The profiles of tecidual coloration had been analyzed through a work station contend an optic microscope copulated with a digital camera, both connected a computer contend software OPTIMAS®. Results: Our results demonstrate a reduction of galectin-3 expression in ulcerative colitis inflammatory site from surgically and clinically treated patients. The goblet cells number from the surgically treated patients had suffered a significant reduction $(p<0.022)$, however with increase of its glandular content. Conclusions: The data suggest that the glandular activity together with the reduction of the galectin-3 expression can assist the accompaniment of these patients.
\end{abstract}

Key words: ulcerative colitis, galectin-3, image digital analises.

\section{REFERÊNCIAS}

01. Rutgeers P - Medical therapy of inflammatory bowel disease. Digestion 1998; 59: p. 453-469

02. Krombluth A, Sachar DK, Salomon P. Crohn's disease. In: Feldman M, Scharscbimdt BF, Sleisenger MH, editors. Sleisenger \& Fordtrans's gastrintestinal and liver disease Pathophysiology: diagnosis and management. 6th ed Philadelphia: W B Saunders; 1998 p. 1708-1734.

03. Robbins LT, Coltron P. Patologia: bases patologias das doenças. 7th.ed. Rio de Janeiro. ABDR. 2005.

04. Nakamura, R M, Matsutani M, Barry M - Advances in Clinical laboratory Tests for Inflammatory Bowel Diseases. Clin Chim Acta 2003; 335: p. 9-20.

05. Chambers et al. - Cancer surveillance in ulcerative colitis. Br J Surg 2005; 92 (8): p. 928-936.

06. Malcomson R D G, McGregor A H - Molecular Screening for Colon cancer in Inflammatory Bowel Disease. Eur J Gastroenterol Hepatol 2002; 14 (10): p. 1045-1047.

07. Rutter M. et al. - Severity of Inflammation is a Risk Factor for Colorectal Neoplasia in Ulcerative Colitis. Gastroenterology 2004; 126: p. 451-459.

08. McDonald S A C et al. - Mechanisms of Disease: from Stem Cells to Colorectal Cancer. Nat Clin Pract Gastroenterol Hepatol 2006; 3 (5): 267-274.

09. Jess T et al. - Risk of Intestinal Cancer in Inflammatory Bowel Disease: A Population-Based Study From Olmsted County, Minnesota. Gastroenterology 2006; 130: p. 1039-1045.

10. Triantafillds J K, Emmanouilidis A, Manousos O N, Nicolakis D, Kovevinas M - Clinical patterns of Crohn's disease in Greece: a follow up study of 155 cases. Digestion 2000; 61: p. 121-128.

11. Irvine E, Faltokbyar F, Swarbrick E T - A critical review of epidemiology studies in inflammatory bowel disease. Scand J Gastroenterol 2001; 36: p. 2-15.
12. Smithson J E, Campbell A, Andrews J M, Milton J D, Pigott R, Jewell D P - Altered expression of mucins throughout the colon in ulcerative colitis. Gut 1997; 40: p. 234-40.

13. Iwasaki A, Medzhitov R - Toll-like receptor control of the adaptive immune responses. Nat Immunol 2004; 5: p. $987-$ 95.

14. Baumgart, D C, Carding S R - Inflammatory bowel disease: cause and immunobiology. Lancet 2007; 369: p. 1627-1640.

15. Gramlich T, Petras R E - Pathology of inflammatory bowel disease. Semin Pediatr Surg 2007; 16: p. 154-163.

16. Eaden J A, Abrams K R, Mayberry J F - The risk of colorectal cancer in ulcerative colitis: a meta-analysis. Gut 2001; 48: p. 526-35.

17. Diaz N J, Patrício F S, Fagundes-Neto U - Colite alérgica: características clínicas e morfológicas da mucosa retal em lactentes com enterorragia. Arq Gastroenterol 2002; 39(4): p. 260-267.

18. Abdel-Hady M, Bunn S K - Inflammatory bowel disease. Cur Pediat 2004; 14(7): p. 598-604.

19. Hughes R C-Galectins. Encyclopedia of Biological Chemistry. Elsevier 2004; 2: p. 171-174.

20. Dumic J, Dabelic S, Flögel M - Galectin-3: an open-ended story. Biochim Biophys Acta 2006; 1760: p. 616-635.

21. Danguy A, Camby I, Robert K - Galectins and Cancer. Bichimi Biophys Acta 2002; 1572: p. 285-293.

22. Francis I M - Manual versus image analysis estimation of PCNA in breast carcinoma. Anal Quant Cytol Histol 2000; 22(1): p. 11-16.

23. Roels S L M F - DNA ploidy and nuclear morphometric variables for the evaluation of melanocytic tumors in dogs and cats. Am J Vet Res 2000; 61(9): p. 1074-1079,

24. Lee C M, Lee R J, Hammond E, Tsodikov A, Dodson M, Zempolich K, Gaffney D K - Expression of HER2neu (cerbB2) epidermal growth factor receptor in cervical cancer: prognostic correlation with clinical characteristics, and 
comparison of manual and automated imaging analysis. Gynecol Oncology 2004; 93: p. 209-214.

25. Rodolfo O, Adhemar L F, Alfredo S, Roberta M, Roberto P, Paola C et al - Nuclear changes in the normal-looking columnar epithelium adjacent to and distant from prostatic intraepithelial neoplasia and prostate cancer. Morphometric analysis in whole-mount sections. Virchows Arch . 2000; 437 (6): p. 62534.

26. Hsu S M, Raine L, Fnager H - Use of avidin-biotin-peroxidase complex $(\mathrm{ABC})$ in immunoperoxidase techniques: $\mathrm{A}$ comparison between $\mathrm{ABC}$ and unlabeled antibody (PAP) procedures. J Histochem Cytochem 1981; 29: p. 577-580.

27. Spicer SS. Histochemistry in pathologic diagnosis. 1st ed New York: Dekker, 1987.

28. Rubinstein N, Ilarregui J M, Toscano M A, Rabinovich G A The role galectins in the initiation, amplification and resolution of the inflammatory response. Tissue Antigens, 2004; 64: p. $1-12$.

29. Lippert E, Gunckel M, Brenmoehl J, Bataille F, Falk W, Scholmerich $\mathrm{J}$ et al. - Regulation of galectin-3 function in mucosal fibroblasts: potential role in mucosal inflammation. Clin Exp Immunol 2008; 152: p. 285-297.

30. Gupta R B, Harpaz N, Itzkowitz S, Hossain S, Matula S, Kornbluth A et al.-Histologic Inflammation Is a Risk Factor for Progression to Colorectal Neoplasia in Ulcerative Colitis: A Cohort Study. Gastroenterology 2007; 133: p. 1099-1005.

31. Sanjuan X, Ferñandez P L, Castells A, Castronovo V, Van Den Brule F, Liu F T et al. - Differential Expression of Galectin 3 and Galectin 1 in Colorectal Cancer Progression. Gastroenterology 1997; 113: p. 1906-1915.

32. Ho G T, Lees C, Satsang J - Ulcerative colitis. Medicine 2007; 35(5): p. 277-282.

\section{Endereço para correspondência:}

GEORGE LUIZ DE SOUZA ARAÚJO

Prédio da Pós-Graduação do Centro de Ciências da Saúde, Av. Prof. Moraes Rego, s/n - Cidade Universitária, 50670-901 - Recife - PE

FAX: (81) 2126-8529

E-mail: george.biomed@universiabrasil.net 